Proyecciones Journal of Mathematics

Vol. 30, $\mathrm{N}^{o}$ 2, pp. 163-174, August 2011.

Universidad Católica del Norte

Antofagasta - Chile

\title{
Partial trees in weighted graphs-I
}

\author{
SUNIL MATHEW \\ NATIONAL INSTITUTE OF TECHNOLOGY, INDIA
}

Received : January 2011. Accepted : May 2011

\begin{abstract}
This paper generalizes the tree concept in Graph Theory, which plays a crucial role in many areas of science and technology. This paper also characterizes partial trees using the concept of maximum spanning trees.
\end{abstract}

Keywords : strength of connectedness, partial tree, partial bridge.

AMS classification : 05C22, 05C38, 05C40 


\section{Introduction}

Weighted graph theory has numerous applications in various fields like clustering analysis, operations research, database theory, network analysis, information theory, etc. Connectivity concepts play a key role in applications related with graphs and weighted graphs. Several authors including Bondy and Fan [2, 3, 4], Broersma, Zhang and Li [13], Sunil Mathew and Sunitha $[8,9,10,11,12]$ introduced many connectivity concepts in weighted graphs following the works of Dirac [6] and Grotschel [7].

In this article we introduce some new connectivity concepts in weighted graphs. In a weighted graph model, for example, in an information network or an electric circuit, the reduction of flow between pairs of nodes is more relevant and may frequently occur than the total disruption of the flow or the disconnection of the entire network [10,11]. Also study of connectivity by levels will be more appropriate in examining the dynamics of the network. This concept is our motivation. As weighted graphs are generalized structures of graphs, the concept introduced in this article also generalizes the classic tree structure in Graph Theory.

A weighted graph $G$ is a graph in which every $\operatorname{arc} e$ is assigned a nonnegative number $w(e)$, called the weight of $e$. The set of all the neighbors of a node $v$ in $G$ is denoted by $N_{G}(v)$ or simply $N(v)$, and its cardinality by $d_{G}(v)$ or $d(v)$ [5]. The weighted degree of $v$ is defined as $d_{G}^{w}(v)=\sum_{x \in N(v)} w(v x)$. When no confusion occurs, we denote $d_{G}^{w}(v)$ by $d^{w}(v)$. The weight of a cycle is defined as the sum of the weights of its arcs. An unweighted graph can be regarded as a weighted graph in which every $\operatorname{arc} e$ is assigned weight $w(e)=1$. Thus, in an unweighted graph, $d^{w}(v)=d(v)$ for every node $v$, and the weight of a cycle is simply the length of the cycle. An optimal cycle is a cycle which has maximum weight $[2]$.

\section{Basic Concepts}

In a weighted graph $G$, we can associate to each pair of nodes in $G$, a real number called strength of connectedness. It is evaluated using strengths of different paths joining the given pair of nodes. We have a set of new definitions which are given below. 
Definition 1: [11] Let $G$ be a weighted graph. The strength of a path $P$ (respectively, strength of a cycle $C$ ) of $n \operatorname{arcs} e_{i}$, for $1 \leq i \leq n$, denoted by $s(P)$ (respectively, $s(C)$ ), is equal to $s(P)=\min _{1 \leq i \leq n}\left\{w\left(e_{i}\right)\right\}$.

Definition 2: [10] Let $G$ be a weighted graph. The strength of connectedness of a pair of nodes $u, v \in V(G)$, denoted by $C O N N_{G}(u, v)$ is defined as $C O N N_{G}(u, v)=\operatorname{Max}\{s(P): P$ is a $u-v$ path in $G\}$. If $u$ and $v$ are in different components of $G$, then $C O N N_{G}(u, v)=0$.

Example 1: Consider the following graph.

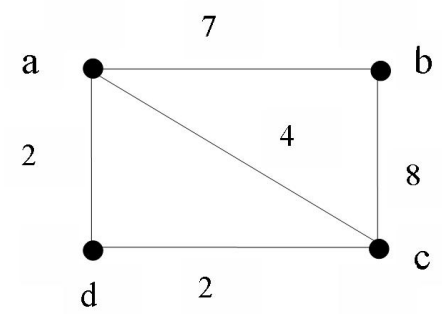

In this graph, $C O N N_{G}(a, b)=7, C O N N_{G}(a, c)=7, C O N N_{G}(a, d)=$ $2, C O N N_{G}(b, c)=8, C O N N_{G}(b, d)=2, C O N N_{G}(c, d)=2$.

Next we have an obvious result.

Proposition 1: [10] Let $G$ be a weighted graph and $H$, a weighted subgraph of $G$. Then for any pair of nodes $u, v \in G$, we have $C O N N_{H}(u, v) \leq$ $C O N N_{G}(u, v)$.

Definition 3: [10] A $u-v$ path in a weighted graph $G$ is called a strongest $u-v$ path if $s(P)=C O N N_{G}(u, v)$.

Definition 4: [10] Let $G$ be a weighted graph. A node $w$ is called a partial cutnode ( $p$-cutnode for short) of $G$ if there exists a pair of nodes $u, v$ in $G$ such that $u \neq v \neq w$ and $C O N N_{G-w}(u, v)<C O N N_{G}(u, v)$. A graph without partial cutnodes is called a partial block (p-block for short) 
It is proved that [10] a node $w$ in a weighted graph $G$ is a $p$-cutnode if and only if $w$ is an internal node of every maximum spanning tree.

Example 2: The following are examples of a $p$-block and a non $p$-block. In fig1 no node is a $p$-cutnode. But in fig 2 , node $u$ is a $p$-cutnode.

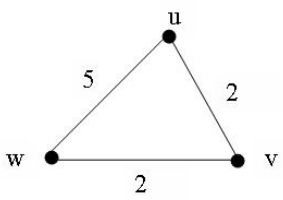

A p-block

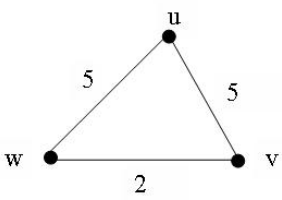

Not a p-block

Definition 5: [10][8] Let $G$ be a weighted graph. An arc $e=(u, v)$ is called a partial bridge ( $p$-bridge for short) if $C O N N_{G-e}(u, v)<C O N N_{G}(u, v)$. A $p$-bridge is said to be a partial bond (p-bond for short) if $C O N N_{G-e}(x, y)<$ $C O N N_{G}(x, y)$ with at least one of $x$ or $y$ different from both $u$ and $v$ and is said to be a partial cutbond (p-cutbond for short) if both $x$ and $y$ are different from $u$ and $v$.

Partial bridges are characterized in [10] and partial bonds and cutbonds in $[8]$.

Example 3: Partial bonds and cut bonds are illustrated in the following example.

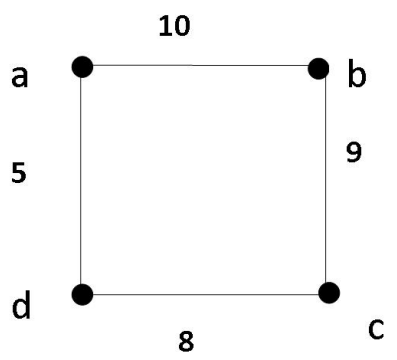


Let $G(V, E)$ be a weighted graph with $V=\{a, b, c, d\}$ and $E=\left\{e_{1}=\right.$ $\left.(a, b), e_{2}=(b, c), e_{3}=(c, d), e_{4}=(d, a)\right\}$ with $w\left(e_{1}\right)=10, w\left(e_{2}\right)=9, w\left(e_{3}\right)=$ $8, w\left(e_{4}\right)=5$. Here all arcs except arc $(a, d)$ are partial bonds. In particular arc $(b, c)$ is a partial cutbond since $C O N N_{G-(b, c)}(a, d)=5<8=$ $C O N N_{G}(a, d)$.

Proposition 2: [10] Let $G$ be a weighted graph and let $w$ be a node in $G$. Then $w$ is a $p$-cutnode if and only if $w$ is an internal node in every strongest $x-y$ path for some pair of nodes $x, y \in V(G)$ other than $w$.

Proposition 3: [10] Let $G$ be a weighted graph and let $e$ be an $\operatorname{arc}$ in $G$. Then $e$ is a $p$-bridge if and only if $e$ is an arc in every strongest $x-y$ path for some pair of nodes $x, y \in V(G)$.

Theorem 1: [10]Let $G$ be a weighted graph and $e=(x, y) \in E(G)$. Then the following statements are equivalent.

(i) $e$ is a $p$-bridge.

(ii) $\operatorname{CONN}_{G-e}(x, y)<w(e)$.

(iii) $e$ is not a weakest arc of any cycle in $G$.

\section{Partial trees in Weighted Graphs}

In this section we introduce a new type of weighted graph, which is not a tree, but at least one of it's threshold subgraphs will be a tree. Note that when $G$ is a weighted graph, its threshold subgraphs need not be trees or forests.

Definition 6: A connected weighted graph $G(V, E)$ is called a weighted partial tree (partial tree for short) if $G$ has a spanning subgraph $F\left(V, E^{\prime}\right)$ which is a tree, where for all $\operatorname{arcs}(x, y)$ of $G$ which are not in $F$, we have $C O N N_{G}(x, y)>w(x, y)$.

When the graph $G$ is not connected and the condition is satisfied by all components of $G$, then $G$ is called partial forest.

Example 4: The following is an example of a partial tree. By removing the arc $(a, b)$ we will get the spanning tree $F$. Note that for arc $(a, b)$, $\operatorname{CONN}_{G}(a, b)=3>w(a, b)$. 


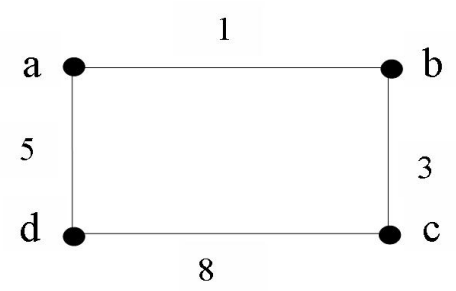

Any weighted tree $T$ is a partial tree. But the converse is not be true as seen from this example.

Next we have a characterization of partial trees.

Theorem 2: A connected weighted graph $G$ is a partial tree if and only if in any cycle $C$ of $G$, there exists an $\operatorname{arc} e=(x, y)$ such that $w(e)<$ $C O N N_{G-e}(x, y)$, where $G-e$ is the subgraph of $G$ obtained by deleting the arc $e$ from $G$.

Proof. Let $G$ be a connected weighted graph. If there are no cycles, it is clearly a tree and hence is a partial tree. If there exists cycles in $G$, let $(x, y)$ be an arc belonging to a cycle $C$ with the minimum weight in $G$. Delete the $\operatorname{arc}(x, y)$ from $G$. If there are still cycles in the graph, we can repeat the process. Now at each stage no previously deleted arc is stronger than the arc being currently deleted. When no cycle remain, the resulting subgraph is a tree $F$. Let $(x, y)$ not be an arc of $F$. Then $(x, y)$ is one of the arcs deleted in the process of constructing $F$. Since $F$ is a tree and the weight of $(x, y)$ was minimum from the arcs of a cycle in $G$, it follows that there exists a path from $x$ to $y$ stronger than $w(x, y)$ and that does not involve $(x, y)$ or any arcs deleted prior to it. If this path involves arcs that were deleted later, the path can be further diverted and so on. This process stabilizes with a path consisting entirely of $\operatorname{arcs}$ of $F$. Thus $G$ is a partial tree.

Conversely, if $G$ is a partial tree and $P$ is any cycle, then some arc $e=(x, y)$ of $P$ does not belong to $F$. Thus by definition we have $w(e)<C O N N_{G-e}(x, y) \leq C O N N_{G}(x, y)$. 
Now we give a sufficient condition for a weighted graph to be a partial tree using the concept of strongest paths.

Theorem 3: If there exist at most one strongest path between any two nodes of $G$, then $G$ must be a partial forest.

Proof. Suppose $G$ is not a partial forest. Then by theorem 1, there is a cycle $C$ in $G$ such that $w(x, y) \geq C O N N_{G}(x, y)$ for all arcs $(x, y)$ of cycle $C$. Thus $(x, y)$ is a strongest path from $x$ to $y$. If we choose $(x, y)$ to be a weakest $\operatorname{arc}$ of $C$, it follows that the rest of the cycle $C$ is also a strongest path from $x$ to $y$, a contradiction.

Theorem 4: If $z$ is a common node of at least two $p$-bridges, then $z$ is a $p$-cutnode.

Proof. Let $\left(u_{1}, z\right)$ and $\left(z, u_{2}\right)$ be two partial bridges. Then by proposition 3 of [10], there exists some $u, v$ such that $\left(u_{1}, z\right)$ is on every strongest $u-v$ path. If $z$ is distinct from $u$ and $v$, it follows that $z$ is a $p$-cutnode. Now suppose that one of $v$ or $u$ is $w$ so that $\left(u_{1}, w\right)$ is on every strongest $u-z$ path or $\left(z, u_{2}\right)$ is on every strongest $w-v$ path. Suppose that $z$ is not a $p$-cutnode. Thus between every two nodes there exists at least one strongest path not containing $z$. In particular, there exists at least one strongest path $P$ joining $u_{1}$ and $u_{2}$ not containing $z$. This path together with $\left(u_{1}, z\right)$ and $\left(z, u_{2}\right)$ forms a cycle.

We now consider two cases. First suppose that $u_{1}, z, u_{2}$ is a not a strongest path. Then one of $\left(u_{1}, z\right),\left(z, u_{2}\right)$ or both becomes the weakest arcs of the the cycle which contradicts that $\left(u_{1}, z\right)$ and $\left(z, u_{2}\right)$ are $p$-bridges.

Second suppose that $u_{1}, z, u_{2}$ is also a strongest path joining $u_{1}$ to $u_{2}$. Then $C O N N_{G}\left(u_{1}, u_{2}\right)=\operatorname{Min}\left\{w\left(u_{1}, z\right), w\left(z, u_{2}\right)\right\}$, the strength of $P$. Thus arcs of $P$ are at least as strong as $w\left(u_{1}, z\right)$ and $w\left(z, u_{2}\right)$ which implies that $\left(u_{1}, z\right)$ and $\left(z, u_{2}\right)$ or both are the weakest arcs of a cycle, which again is a contradiction. ( By theorem 1)

The condition in the above theorem is not necessary as as seen from the following example. 


\section{Example 5:}

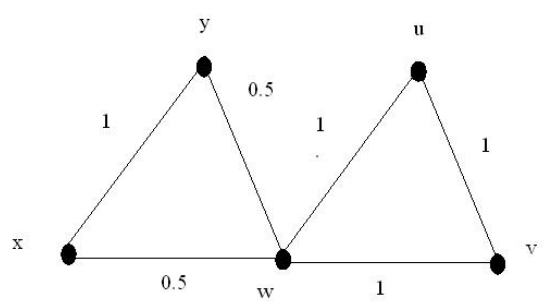

In this graph, node $w$ is a cutnode and hence is a $p$-cutnode, but it is not a common node of two or more $p$-bridges.

Theorem 5: Let $G$ be a weighted graph. If $e=(u, v)$ is a $p$-bridge in $G$, then $C O N N_{G}(u, v)=w(e)$.

Proof. Suppose that $(u, v)$ is a bridge and that $C O N N_{G}(u, v)>w(e)$. Then there exists a strongest $u-v$ path with strength greater than $w(u, v)$ and all arcs of this strongest path have strength greater than $w(u, v)$. Now this path together with the arc $(u, v)$ forms a cycle in which $(u, v)$ is the weakest arc, contradicting that $(u, v)$ is a $p$-bridge.

The converse of Theorem 5 is not true. The condition for the converse to be true is discussed in Theorem 9 .

Theorem 6: If $G$ is a weighted partial tree and is not a tree, then there exists at least one arc $(u, v)$ for which $w(u, v)<C O N N_{G}(u, v)$.

Proof. If $G$ is a partial tree, then by definition there exists a spanning tree $F$ such that $w(u, v)<C O N N_{G}(u, v)$ for all $\operatorname{arcs}(u, v)$ not in $F$. By hypothesis, there exists at least one such arc (since $G$ is not a tree) and the result follows.

The concept of partial cutnodes and partial bridges are introduced in [11]. Next theorem gives the information about the partial cutnodes of a weighted partial tree. 
Theorem 7: If $G$ is a partial tree and $F$, the spanning tree in the definition, then the arcs of $F$ are the partial bridges of $G$.

Proof. Let $(u, v)$ be an arc in $F$. Then this arc is the unique path between $u$ and $v$ in $F$. If there is no other paths in $G$ from $u$ to $v$, then clearly $(u, v)$ is a bridge of $G$ and hence is a partial bridge of $G$. If there exists a path say $P$ from $u$ to $v$ in $G$, then $P$ will definitely contain an arc $(x, y)$ which is not in $F$ such that $C O N N_{G}(x, y)>w(x, y)$. Then $(u, v)$ is not a weakest arc of any cycle in $G$ and hence by Theorem $1,(u, v)$ is a partial bridge.

Theorem 8: If $G$ is a partial tree and $F$ the spanning tree with the property given in definition, then the internal nodes of $F$ are the partial cutnodes of $G$.

Proof. Let $z$ be any node in $G$ which is not a pendent node of $F$. Then $z$ is the common node of two at least two $\operatorname{arcs}$ of $F$, which are bridges of $G$. By Theorem $4, z$ is a $p$-cutnode. Also if $z$ is a pendant node of $F$, then $z$ is a not a $p$-cutnode; else there would exist $u, v$ different from $z$ such that $z$ is on every strongest strongest $u-v$ path and one such path certainly lies in $F$. But since $z$ is a pendant node of $F$, this is impossible.

Theorem 9: $G$ is a weighted partial tree if and only if the following are equivalent for all $u, v$ :

(i) $(u, v)$ is a $p$-bridge.

(ii) $C O N N_{G}(u, v)=w(u, v)$.

Proof. Let $G$ be a partial tree and suppose that $e=(u, v)$ is a $p$-bridge. Then by Theorem $5, C O N N_{G}(u, v)=w(e)$. Now let $(u, v)$ be an $\operatorname{arc}$ in $G$ such that $C O N N_{G}(u, v)=w(e)$. If the underlying graph (graphs obtained by removing weights) is a tree, then clearly $(u, v)$ is a bridge and hence is a $p$-bridge. If the underlying graph is not a tree, then by Theorem $6,(u, v)$ is in $F$ and hence $(u, v)$ is a $p$-bridge.

Conversely assume that (i) and (ii) are equivalent. Construct a maximum spanning tree $T$ for $G$. If $(u, v)$ is in $T$, by an algorithm in [1], $C O N N_{G}(u, v)=w(u, v)$ and hence $(u, v)$ is a $p$-bridge. Now these are the 
only $p$-bridges of $G$; for if possible let $\left(u^{\prime}, v^{\prime}\right)$ be a $p$-bridge of $G$ which is not in $T$. Consider a cycle $C$ consisting of $\left(u^{\prime}, v^{\prime}\right)$ and the unique $u^{\prime}-v^{\prime}$ path in $T$. Now arcs of this $u^{\prime}-v^{\prime}$ path are $p$-bridges and so they are not weakest $\operatorname{arcs}$ of $C[10]$ and thus $\left(u^{\prime}, v^{\prime}\right)$ must be the weakest arc of $C$ and thus cannot be a bridge.

Moreover, for all $\operatorname{arcs}\left(u^{\prime}, v^{\prime}\right)$ not in $T$, we have $w\left(u^{\prime}, v^{\prime}\right)<C O N N_{T}\left(u^{\prime}, v^{\prime}\right)$; for if possible let $w\left(u^{\prime}, v^{\prime}\right) \geq C O N N_{T}\left(u^{\prime}, v^{\prime}\right)$. But $w\left(u^{\prime}, v^{\prime}\right)<C O N N_{G}\left(u^{\prime}, v^{\prime}\right)$ where strict inequality holds since $\left(u^{\prime}, v^{\prime}\right)$ is not a $p$-bridge. Hence $\operatorname{CONNT}\left(u^{\prime}, v^{\prime}\right)<C O N N_{G}\left(u^{\prime}, v^{\prime}\right)$ which gives a contradiction since $C O N N_{T}\left(u^{\prime}, v^{\prime}\right)$ is the strength of the unique $u^{\prime}-v^{\prime}$ path in $T$ and by an algorithm in [1], CONN $N_{G}\left(u^{\prime}, v^{\prime}\right)=C O N N_{T}\left(u^{\prime}, v^{\prime}\right)$. Thus $T$ is the required spanning subgraph $F$, which is a tree and hence $G$ is a partial tree.

For a partial tree the spanning subgraph $F$ is unique. It follows from the proof of Theorem 8 that $F$ is nothing but the maximum spanning tree of $G$. Thus we have the following theorem.

Theorem 10: A weighted graph is a partial tree if and only if it has a unique maximum spanning tree.

\section{Concluding remarks}

Connectivity concepts are the key in graph clustering and network problems. The classical parameters are dealing with the disconnection of the graph. In practical applications the reduction in the flow is more frequent than the disconnection. The authors made an attempt to generalize the concept of trees in weighted graphs.

\section{Acknowledgements}

This work is supported by National Institute of Technology Calicut, India under FRG scheme. We thank the authorities for the sanctioning of the project and giving further assistance. 


\section{References}

[1] P. Bhattacharya, suraweera, An algorithm to compute the supremum of max-min powers and a property of fuzzy graphs, Pattern Recognition Letters, 12, pp.413-420, (1987).

[2] J. A.Bondy, G. Fan, Optimal paths and cycles in weighted graphs, Ann.Discrete Mathematics, 41, pp.53-69, (1989).

[3] J. A. Bondy, G. Fan, Cycles in weighted graphs, Combinatorica, 11(1991), pp. 191-205, (1991).

[4] J. A. Bondy, H. J. Broersma, J. van den Heuvel and H. J. Veldman, Heavy cycles in weighted graphs, Discuss. Math. Graph Theory, 22, pp. $7-15,(2002)$.

[5] R. Diestel, Graph Theory, Second edition, Graduate texts in mathematics 173, Springer, (2000).

[6] G. A. Dirac, Some theorems on abstract graphs, Proc. London Math. Soc., (3) 2, pp. 69 - 81, (1952).

[7] M. Grotschel, Graphs with cycles containing given paths, Ann. Discrete Math., 1, pp.233 - 245, (1977).

[8] Sunil Mathew, M.S.Sunitha, Bonds in graphs and fuzzy graphs, Advances in Fuzzy Sets and Systems, 6 (2), pp. 107-119, (2010).

[9] Sunil Mathew, M. S. Sunitha, Node connectivity and arc connectivity of a fuzzy graph, Information Sciences 180, pp. 519-531, (2010).

[10] Sunil Mathew, M. S. Sunitha, Some connectivity concepts in weighted graphs, Advances and Applications in Discrete Mathematics 6 (1), pp. 45-54, (2010).

[11] Sunil Mathew, M. S. Sunitha, Types of arcs in a fuzzy graph, Information Sciences, 179(11), pp. 1760-1768, (2009).

[12] Sunil Mathew, M. S. Sunitha, Cycle connectivity in weighted graphs, Proyecciones Journal of Mathematics, 30, 1, pp. 1-17, (2009). 
[13] S. Zang, X. Li,H. Broersma, Heavy paths and cycles in weighted graphs, Discrete Math., 223, pp. 327-336, (2000).

\section{Sunil Mathew}

Department of Mathematics

National Institute of Technology Calicut

Calicut - 673601

India

e-mail : sm@nitc.ac.in 